BMJ Open

Diabetes

Research

\& Care

\title{
Association between varying cut-points of intermediate hyperglycemia and risk of mortality, cardiovascular events and chronic kidney disease: a systematic review and meta-analysis
}

\author{
Unjali P Gujral (10 , ${ }^{1}$ Ram Jagannathan, ${ }^{2}$ Siran $\mathrm{He},{ }^{3}$ Minxuan Huang, ${ }^{4}$ \\ Lisa R Staimez (D) , ${ }^{1}$ Jingkai Wei, ${ }^{5}$ Nanki Singh, ${ }^{6}$ K M Venkat Narayan (D) ${ }^{1}$
}

To cite: Gujral UP, Jagannathan $\mathrm{R}$, He S, et al. Association between varying cut-points of intermediate hyperglycemia and risk of mortality, cardiovascular events and chronic kidney disease: a systematic review and meta-analysis. BMJ Open Diab Res Care 2021;9:e001776. doi:10.1136/ bmjdrc-2020-001776

- Supplemental material is published online only. To view please visit the journal online (http://dx.doi.org/10.1136/ bmjdrc-2020-001776).

Received 15 July 2020 Revised 15 February 2021 Accepted 8 March 2021
Check for updates

(c) Author(s) (or their employer(s)) 2021. Re-use permitted under CC BY-NC. No commercial re-use. See rights and permissions. Published by BMJ.

For numbered affiliations see end of article.

Correspondence to Dr Unjali P Gujral; ugujral@emory.edu

\section{ABSTRACT}

Introduction We conducted a systematic review and meta-analysis to evaluate the updated evidence regarding prediabetes for predicting mortality, macrovascular and microvascular outcomes.

Research design and methods We identified English language studies from MEDLINE, PubMed, OVID and Cochrane database indexed from inception to January 31,2020 . Paired reviewers independently identified 106 prospective studies, comprising nearly 1.85 million people, from 27 countries. Primary outcomes were allcause mortality (ACM), cardiovascular mortality (CVDM), cardiovascular disease (CVD), coronary heart disease (CHD) and stroke. Secondary outcomes were heart failure, chronic kidney disease (CKD) and retinopathy.

Results Impaired glucose tolerance was associated with ACM; HR 1.19, 95\% Cl (1.15 to 1.24), CVDM; HR 1.21, $95 \% \mathrm{Cl}$ (1.10 to 1.32), CVD; HR 1.18, 95\% Cl (1.11 to 1.26$)$, CHD; HR; $1.13,95 \% \mathrm{Cl}$ (1.05 to 1.21) and stroke; HR 1.24, $95 \% \mathrm{Cl}$ (1.06 to 1.45). Impaired fasting glucose (IFG) 110$125 \mathrm{mg} / \mathrm{dL}$ was associated with ACM; HR $1.17,95 \% \mathrm{Cl}$ (1.13 to 1.22), CVDM; HR $1.20,95 \% \mathrm{Cl}$ (1.09 to 1.33), CVD; HR $1.21,95 \% \mathrm{Cl}$ (1.09 to 1.33), CHD; HR; $1.14,95 \% \mathrm{Cl}$ (1.06 to 1.22) and stroke; HR $1.22,95 \% \mathrm{Cl}(1.07$ to 1.40$)$. IFG 100-125 mg/dL was associated with ACM; HR 1.11, $95 \% \mathrm{Cl}$ (1.04 to 1.19), CVDM; HR $1.14,95 \% \mathrm{Cl}$ (1.03 to 1.25), CVD; HR 1.15, 95\% Cl (1.05 to 1.25), CHD HR; 1.10 , $95 \% \mathrm{Cl}(1.02$ to 1.19$)$ and $\mathrm{CKD} ; \mathrm{HR} ; 1.09,95 \% \mathrm{Cl}(1.01$ to 1.18). Glycosylated hemoglobin $\mathrm{A} 1 \mathrm{c}(\mathrm{HbA} 1 \mathrm{c}) 6.0 \%-6.4 \%$ was associated with $\mathrm{ACM}$; HR $1.30,95 \% \mathrm{Cl}(1.03$ to 1.66$)$, CVD; HR 1.32, 95\% Cl (1.00 to 1.73) and CKD; HR 1.50, $95 \% \mathrm{Cl}$ (1.32 to 1.70). HbA1c $5.7 \%-6.4 \%$ was associated with CVD HR 1.15, 95\% Cl (1.02 to 1.30), CHD; HR 1.28, $95 \% \mathrm{Cl}$ (1.13 to 1.46), stroke; HR $1.23,95 \% \mathrm{Cl}$ (1.04 to 1.46) and CKD; HR 1.32, 95\% $\mathrm{Cl}$ (1.16 to 1.50$)$. Conclusion Prediabetes is an elevated risk state for macrovascular and microvascular outcomes. The prevention and management of prediabetes should be considered.

\section{INTRODUCTION}

Elevated blood glucose concentrations, such as impaired fasting glucose (IFG), impaired

\section{Significance of this study}

What is already known about this subject?

- WHO and the American Diabetes Associaiton (ADA) differ on the definitions of the lower thresholds of impaired fasting glucose and prediabetic glycosylated hemoglobin A1c (HbA1c).

- Prediabetes is associated with increased risk for type 2 diabetes.

- There is evidence to indicate that impaired glucose tolerance and impaired fasting glucose are also associated with adverse mortality and cardiovascular outcomes.

What are the new findings?

- Impaired glucose tolerance, impaired fasting glucose and elevated $\mathrm{HbA} 1 \mathrm{c}$ are associated with and increased risk of mortality, cardiovascular outcomes and chroic kidney disease.

- Elevated $\mathrm{HbA1C}$ is associated with an icreased risk of both macrovascular and microvascular outcomes.

- The lower cut-point of intermediate HbA1c was associated with increased cardiovascular events while the higher cut-point was associated with increased mortality.

- Both HbA1c cut-points as well as impaired fasting glucose ADA were associated with increased risk of incident chronic kidney disease.

How might these results change the focus of research or clinical practice?

- Prediabetes is an elevated risk state for adverse events beyond type 2 diabetes, and the prevention and management of prediabetes including elevated $\mathrm{HbA1c}$ should be considered.

glucose tolerance (IGT) and elevated glycosylated hemoglobin A1c (HbAlc), represent stages of glycemia considered too high to be in the normal range, but below the threshold of what is considered type 2 diabetes. ${ }^{1}$ While elevated glucose poses an increased risk for 


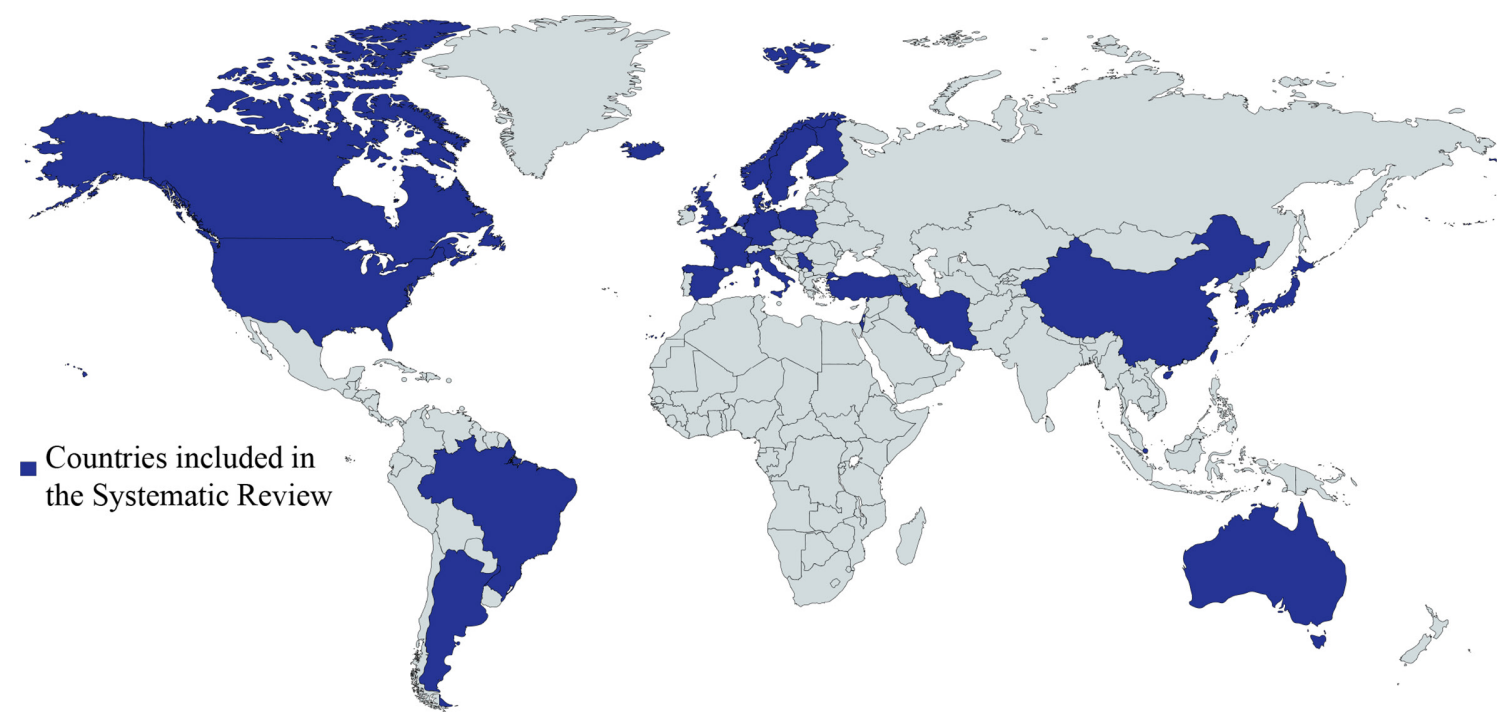

Figure 1 Countries included in the systematic review. Number of cohorts including each country: Argentina: 1; Australia: 3; Brazil: 1; Canada: 1; China: 10; Denmark: 3; Fiji: 1; Finland: 7; France: 1; Germany: 4; Iceland: 2; Iran: 2; Israel: 2; Italy: 3; Japan: 10; Mauritius: 2; The Netherlands: 4; Norway: 2; Poland: 1; Serbia: 1; Singapore: 2; South Korea: 6; Spain: 1; Sweden: 2; Turkey: 1; UK: 8; USA: 23.

type 2 diabetes, individuals in this intermediate range may also be at increased risk for all-cause mortality, cardiovascular mortality, incident cardiovascular events and microvascular complications. Importantly, the American Diabetes Association (ADA) and WHO currently differ on how they define the lower threshold for IFG as well as identifying an intermediate stage of $\mathrm{HbAlc}$. While WHO defines IFG as fasting glycemia between 110 and $125 \mathrm{mg} / \mathrm{dL}^{2}$ the ADA has reduced the lower threshold to $100 \mathrm{mg} / \mathrm{dL}{ }^{1}$ Furthermore, WHO does not recommend HbAlc as a suitable test for the diagnosis of intermediate glycemia. ${ }^{3}$ However, the ADA suggests a cut-point of HbAlc $5.7 \%-6.4 \%$ for intermediate hyperglycemia, while an International Expert Committee (IEC) ${ }^{4}$ as well as the UK-based National Institute for Health and Clinical Excellence $^{5}$ both recommend using HbA1c 6.0\%-6.4\%

In addition, IFG, IGT and elevated HbAlc represent different types of hyperglycemia, possibly varying in pathophysiological mechanisms. ${ }^{67}$ For example, insulin resistance may be the primary defect in individuals with isolated IGT, while dysfunction in insulin secretion has been seen as the earliest observed defect in individuals with IFG. ${ }^{6}$ It is important to investigate if the varying forms of intermediate hyperglycemia predict a different risk spectrum for developing mortality, cardiovascular disease (CVD) or microvascular complications. We, therefore, conducted a systematic review and meta-analysis of 106 prospective studies, comprising nearly 1.85 million people, from 27 countries (figure 1), to evaluate the nondiabetic cut-points of fasting glucose, 2-hour glucose and HbAlc for predicting the primary outcomes of all-cause mortality, cardiovascular mortality, cardiovascular events, heart disease events and stroke events. We also considered the secondary outcomes of heart failure, chronic kidney disease and retinopathy.

\section{METHODS}

\section{Data sources and searches}

We searched the electronic databases of Medline, PubMed, OVID and Cochrane for prospective cohort studies up to January 31, 2020. With the assistance of a certified librarian, we developed a search strategy (online supplemental table 1) using a combination of Medical Subject Headings and text search based on the following root terms: "prediabetes", "intermediate hyperglycemia", "impaired fasting glucose", "IFG", "impaired glucose tolerance", "IGT", "HbA1c", "elevated HbA1c", "raised HbA1c", "glycosylated hemoglobin A1c", "complication", "mortality", "cardiovascular disease or CVD", "coronary heart disease or CHD", "heart failure", "heart attack", "myocardial infarction", "angina", "ischemia", "cardiac failure", "cerebrovascular", "revascularization", "cerebral infarction", "peripheral artery disease", "retinopathy", "neuropathy", "polyneuropathy", nephropathy", "kidney or renal disease", "CRD or CRF or CKF or CRF or CKD or ESKD or ESKF or ESRD or ESRF", "microvascular", "macrovascular", "cancer", "neoplasm”, "tumor" and "amputation", "predict", "association", "prognosis", "predictive model", "prognostic model", "predictive value", "risk prediction", "risk factor", "risk score".

\section{Study selection}

We identified 7153 studies for abstract screening. Studies were included for analysis if they met the following criteria: were prospective cohort studies of adult individuals aged 18 years and older, were from the general population or from patients with previous atherosclerotic CVD, had measures of impaired fasting glucose, impaired glucose tolerance or intermediate HbAlc as defined by the ADA, WHO or IEC criteria that was evaluated at baseline, included at least one outcome of interest, and 
reported adjusted HRs, relative risks (RRs) or ORs for the risk of prediabetes and at at least one outcome of interest. Studies that did not have longitudinal measures of prediabetes as well as at least one relevant outcome, were not conducted in humans, and were not published in the English language were excluded from full-text review. A total of 280 studies were included in the full-text review. Of these, 174 studies were excluded for the following reasons; the study did not have a cut-point for intermediate hyperglycemia that was consistent with the WHO, ADA or IEC criteria: the study was an earlier version of a study included in the analysis; the study was a duplicate of another study included in the analysis; the study population had a pre-existing condition aside from atherosclerotic CVD; full-text of the study was not available; no relevant measures of association or CIs were reported; follow-up time was $<2$ years and no relevant outcome was reported. In total, 106 studies were included (online supplemental figure 1). Cohort studies were included multiple times if they assessed the associations between differing glycemic measures and outcomes. However, only the largest sample size of each cohort was counted towards the overall sample size of the analysis. Primary outcomes of interest were all-cause mortality, cardiovascular mortality, cardiovascular events, heart failure events and stroke events. Secondary outcomes of interest were heart failure, chronic kidney disease and retinopathy.

Our study protocol was developed in consensus with Emory investigators. This meta-analysis was not prospectively registered in the PROSPERO database.

\section{Data extraction and quality assessment}

Each title and abstract was screened independently by two reviewers (UPG, MH, SH, LRS, RJ, JW). Discrepancies were resolved by consensus or by a third reviewer. Fulltext articles that met the inclusion criteria were obtained and study information such as sample size, mean age of participants, sex distribution, race/ethnicity, comorbidities, demographic characteristics and the relationship between non-diabetic glucose measures and outcomes were extracted independently by two reviewers on standard forms. The results were compared, and discrepancies were resolved by a third reviewer.

We used the Quality in Prognosis Studies Tool for study quality assessment. Validity and bias were judged on study participation, study attrition, prognostic factor measurement, confounding measurement and account, outcome measurement and analysis and reporting. ${ }^{8}$ Two review authors independently rated the quality of evidence for each outcome (online supplemental table 2).

\section{Data synthesis and analysis}

We extracted the following information from each study: study characteristics (study name, author, publication year, pre-existing cardiovascular condition, location/ region, follow-up time, sample size), participant's characteristics (mean age, gender distribution), primary and secondary outcomes (total mortality, CVD mortality,
CVD events, heart disease events, stroke events, heart failure events, chronic kidney disease events and retinopathy). We also extracted point estimates (HRs, risk ratios or ORs) for the association between prediabetes and outcomes as well as all covariates included in the models. The data were entered into an excel spreadsheet specific for this study with an appropriate data validation feature to control the data type and value and to identify invalid entries. This study's primary purpose was to study the association between the differing definitions of intermediate hyperglycemia and all-cause and cardiovascular mortality, CVD, heart disease and stroke events compared with normoglycemia. CVD events were defined as the occurrence of more than one cardiovascular event. The secondary purpose was to compare the association between the differing definitions of intermediate hyperglycemia and heart failure, chronic kidney disease and retinopathy. The following definitions of intermediate hyperglycemia were used: IGT was defined as $140-199 \mathrm{mg} / \mathrm{dL}$. IFG was defined according to the WHO criteria of $110-125 \mathrm{mg} / \mathrm{dL}$ (IFG WHO) or the ADA criteria of 100-125 mg/dL (IFG ADA). ${ }^{12}$ Elevated HbAlc was defined by either the ADA definition of $5.7 \%-6.4 \%$ (HbA1c ADA) or the IEC definition of $6.0 \%-6.4 \%$ (HbA1c IEC). ${ }^{4}$ Reference levels were normal glycemia as defined by each of the ADA, WHO or IEC criterion.

In this meta-analysis, the HRs and 95\% CIs were appraised as the effect size for all the studies, and HRs were deemed equivalent to RRs. The formula $\mathrm{RR}=\mathrm{OR} /$ $([1-\mathrm{pRef}]+[\mathrm{pRef} \times \mathrm{OR}])$, where pRef is the prevalence of the outcome in the reference group that was used to convert ORs to RRs for analysis. ${ }^{9}{ }^{10}$ Any results stratified by sex or race/ethnicity were handled as separate reports.

Summary HRs using both random-effects and fixedeffects models were obtained with the calculation of the logarithm of the HRs and corresponding $95 \%$ CIs of the individual studies. Forest plots were constructed to visually assess the pooled HRs and corresponding 95\% CIs across studies. We explored the statistical heterogeneity across studies by $\mathrm{I}^{2}$ statistic. Values of $<25 \%$ were considered to represent a low likelihood of differences between studies, with values of $25 \%-75 \%$ representing a moderate likelihood, and those $>75 \%-100 \%$ representing a high likelihood. A Cochran Q-test $\mathrm{p}<0.10$ was considered indicative of statistically significant heterogeneity. ${ }^{11}$ HRs were pooled using the fixed-effect model if no or low heterogeneity was observed. Otherwise, the DerSimonian and Laird random-effects model was used, ${ }^{12}$ and the weights were equal to the inverse variance of each study's effect estimation. Publication bias was evaluated by inspecting funnel plots for each outcome in which the natural log $\mathrm{RR}$ was plotted against the SE and further tested with Egger's tests.

Sensitivity analysis was conducted after the removal of extreme effect sizes and by excluding each study sequentially by the leave-one-out diagnostics to evaluate whether a particular study may have strongly influenced the summary risk estimate. ${ }^{13}$ In addition, a priori 
Table 1 Associations between intermediate hyperglycemia and outcomes of interest

\begin{tabular}{llllll}
\hline & IGT & & IFG & HbA1c & \\
\hline Comparison group & 140 to $199 \mathrm{mg} / \mathrm{dL}$ & 100 to $125 \mathrm{mg} / \mathrm{dL}$ & 110 to $125 \mathrm{mg} / \mathrm{dL}$ & $5.7 \%$ to $6.4 \%$ & $6.0 \%$ to $6.4 \%$ \\
\hline Referent group & $<140 \mathrm{mg} / \mathrm{dL}$ & $<100 \mathrm{mg} / \mathrm{dL}$ & $<110 \mathrm{mg} / \mathrm{dL}$ & $<5.7 \%$ & $<6.0 \%$ \\
\hline All-cause mortality & $1.19(1.15$ to 1.24$)$ & $1.11(1.04$ to 1.19$)$ & $1.17(1.13$ to 1.22$)$ & $1.07(0.97$ to 1.18$)$ & $1.30(1.03$ to 1.66$)$ \\
\hline CVD mortality & $1.21(1.10$ to 1.32$)$ & $1.14(1.03$ to 1.25$)$ & $1.20(1.09$ to 1.33$)$ & $1.20(0.90$ to 1.60$)$ & $1.07(0.61$ to 1.87$)$ \\
\hline CVD events & $1.18(1.11$ to 1.26$)$ & $1.15(1.05$ to 1.25$)$ & $1.21(1.09$ to 1.33$)$ & $1.15(1.02$ to 1.30$)$ & $1.32(1.00$ to 1.73$)$ \\
\hline Stroke events & $1.24(1.06$ to 1.45$)$ & $1.07(0.95$ to 1.07$)$ & $1.22(1.07$ to 1.40$)$ & $1.23(1.04$ to 1.46$)$ & $1.28(0.72$ to 2.27$)$ \\
\hline CHD events & $1.13(1.05$ to 1.21$)$ & $1.10(1.02$ to 1.19$)$ & $1.14(1.06$ to 1.22$)$ & $1.28(1.13$ to 1.46$)$ & $1.33(0.74$ to 2.38$)$ \\
\hline Heart failure events & $5.04(1.00$ to 25.4$)$ & $0.95(0.84$ to 1.07$)$ & - & $1.13(0.87$ to 1.45$)$ & - \\
\hline CKD & $1.15(0.95$ to 1.39$)$ & $1.09(1.01$ to 1.18$)$ & $1.08(0.91$ to 1.28$)$ & $1.32(1.16$ to 1.50$)$ & $1.50(1.32$ to 1.70$)$ \\
\hline Retinopathy & - & $1.11(0.84$ to 1.45$)$ & - & $0.84(0.61$ to 1.14$)$ & - \\
\hline
\end{tabular}

CHD, coronary heart disease; CKD, chronic kidney disease; CVD, cardiovascular disease; HbA1c, glycosylated hemoglobin A1c; IFG, impaired fasting glucose; IGT, impaired glucose tolerance.

subgroup analyses of primary and secondary outcomes were conducted according to region (North America vs others), and with pre-existing baseline conditions (yes/ no).

All meta-analyses were conducted in R (V.3.5.1; R foundation of statistical computing, Vienna, Austria) statistical platform using the packages 'meta' (V.4.9-6) ${ }^{14}$ and 'metafor' (V.2.1-0), ${ }^{15}$ with a two-tailed $\alpha$ of 0.05 considered statistically significant.

\section{RESULTS}

Online supplemental table 3 details the key characteristics of all included studies. A majority of the cohorts came from Europe $(n=39)$, Asia $(n=28)$ and the USA $(n=23)$. The duration of follow-up ranged from 2 to 33 years, with a mean duration of 9.57 years. According to quality assessment criteria (online supplemental table 2), the majority of studies were at low risk of bias.

The associations between intermediate hyperglycemia and the outcomes of interest are detailed in table 1.

\section{Intermediate hyperglycemia and all-cause mortality}

Of the 52 studies reporting the association between hyperglycemia and all-cause mortality, 25 examined the association of all-cause mortality with IGT, 25 examined the association with IFG ADA, 21 examined the association with IFG WHO, 14 examined the association with HbA1c 5.7\%-6.4\% and 3 examined the association with HbA1c $6.0 \%-6.4 \%$. Compared with those with normal glucose tolerance, individuals with IGT; HR $1.19,95 \%$ CI (1.15 to 1.24$)$, IFG WHO; HR $1.17,95 \%$ CI (1.13 to 1.22); IFG ADA; HR 1.11, 95\% CI (1.04 to 1.19$)$ and HbAlc $6.0 \%-6.4 \%$; HR $1.30,95 \%$ CI (1.03 to 1.66 ) had an increased risk for all-cause mortality (online supplemental figure 2). There was no evidence of an increased risk of all-cause mortality and HbA1c 5.7\%-6.4\%.

Online supplemental table 4 details the associations between all-cause mortality and intermediate hyperglycemia by presence of pre-existing CVD and region. There were significant differences in the association of
IFG ADA and IFG WHO and all-cause mortality by presence of pre-existing condition. Those who had a preexisting condition as well as prediabetes defined by the IFG ADA or the IFG WHO criteria had an increased risk of all-cause mortality compared with those in the general population. There were no significant differences in the association between prediabetes and all-cause mortality by pre-existing condition for IGT, HbA1c 6.0\%-6.4\% or HbAlc $5.7 \%-6.4 \%$. There were no significant differences in the association of IFG ADA, IFG WHO or HbAlc $5.7 \%-6.4 \%$ and mortality by region. However, the association between IGT and mortality was increased among studies from Australia and Asia compared with the USA. HbA1c $6.0 \%-6.4 \%$ and mortality was increased among studies from the USA compared with Asia and Europe.

We found slight evidence of publication bias among studies assessing the association between all-cause mortality and intermediate hyperglycemia based on visual inspection of the funnel plot (online supplemental figure 9).

\section{Intermediate hyperglycemia and cardiovascular mortality}

Of the 41 studies reporting the association between intermediate hyperglycemia and cardiovascular mortality, 20 examined the association with IGT, 21 examined the association with IFG ADA, 17 examined the association with IFG WHO, 6 examined the association with HbAlc ADA and 1 examined the association with HbA1c IEC. Compared with those with normal glucose tolerance, individuals with IGT; HR 1.21, 95\% CI (1.10 to 1.32), IFG WHO; HR 1.20, 95\% CI (1.09 to 1.33) and IFG ADA; HR $1.14,95 \%$ CI (1.03 to 1.25$)$ had an increased risk for cardiovascular mortality (online supplemental figure 3). There was no evidence of an increased risk of cardiovascular mortality and HbAlc $5.7 \%-6.4 \%$ or HbAlc $6.0 \%-6.4 \%$.

Those with IFG ADA and pre-existing CVD had an increased risk of cardiovascular mortality compared with those without pre-existing CVD. There were no significant differences in the association between prediabetes and 
cardiovascular mortality by the presence of pre-existing CVD for those with IFG WHO, IGT, HbA1c 5.7\%-6.4\% or HbAlc $6.0 \%-6.4 \%$. There were significant differences in the association between prediabetes and cardiovascular mortality by region. The association between IFG ADA and cardiovascular mortality was increased in cohorts from Europe and Asia compared with those from the USA. The association between HbAlc 5.7\%$6.4 \%$ was increased in studies from the USA compared with Europe and Asia. There were no significant differences in the association between intermediate hyperglycemia and cardiovascular mortality by region using the IFG WHO, IGT or HbAlc 6.0\%-6.4\% cut-points (online supplemental table 4).

We found slight evidence of publication bias among studies assessing the association between cardiovascular mortality and intermediate hyperglycemia based on visual inspection of the funnel plot (online supplemental figure 10).

\section{Intermediate hyperglycemia and incident cardiovascular disease}

Of the 35 studies that reported the association between intermediate hyperglycemia and CVD, 17 examined the association with IGT, 24 examined the association with IFG ADA, 7 examined the association with IFG WHO, 13 examined the association with HbA1c 5.7\%-6.4\% and 4 examined the association with HbA1c 6.0\%-6.4\%. Compared with those with normal glucose tolerance, individuals with IGT; HR 1.18, 95\% CI (1.11 to 1.26), IFG WHO; HR 1.21, 95\% CI (1.09 to 1.33); IFG ADA; HR $1.15,95 \%$ CI (1.05 to 1.25 ); HbA1c $5.7 \%-6.4 \%$; HR 1.15 , $95 \%$ CI (1.02 to 1.30$)$ and HbA1c $6.0 \%-6.4 \%$; HR 1.32 , $95 \%$ CI (1.00 to 1.73 ) had an increased risk for cardiovascular events (online supplemental figure 4).

There were no significant differences in the association between prediabetes and cardiovascular events by presence of a pre-existing CVD. However, there were significant differences in the association between prediabetes and CVD by region among those with HbAlc 6.0\%-6.4\%. The association between CVD and HbA1c 6.0\%-6.4\% cutpoints was increased in studies from the USA compared with those from Europe. There were no differnces in the association of IGT, IFG ADA, IFG WHO and HbAlc $5.7 \%-6.4 \%$ and CVD events by region.

We found no evidence of publication bias among studies assessing the association between CVD events and intermediate hyperglycemia based on visual inspection of the funnel plot (online supplemental figure 11).

\section{Intermediate hyperglycemia and incident stroke}

Of the 24 studies that reported the association between intermediate hyperglycemia and stroke, 10 examined the association with IGT, 15 examined the association with IFG ADA, 6 examined the association with IFG WHO, 5 examined the association with HbA1c $5.7 \%-6.4 \%$ and 1 examined the association with HbAlc 6.0\%-6.4\%. Compared with those with normal glucose tolerance, individuals with IGT; HR 1.24, 95\% CI (1.06 to 1.45$)$, IFG WHO; HR 1.22, 95\% CI (1.07 to 1.40 ) and HbA1c $5.7 \%$ 6.4\%; HR 1.23 95\% CI (1.04 to 1.46 ) had an increased risk for stroke. There was no evidence of an increased risk of stroke and IFG ADA or HbA1c 6.0\%-6.4\% (online supplemental figure 5).

There were no significant differences in the association between prediabetes and incident stroke by presence of pre-existing CVD or geographic region.

We found moderate evidence of publication bias among studies assessing the association between incident stroke and intermediate hyperglycemia based on visual inspection of the funnel plot (online supplemental figure 12).

\section{Intermediate hyperglycemia and incident heart disease}

Of the 27 studies that reported the association between intermediate hyperglycemia and heart disease, 12 examined the association with IGT, 16 examined the association with IFG ADA, 9 examined the association with IFG WHO, 4 examined the association with HbAlc $5.7 \%-6.4 \%$ and 1 examined the association with $\mathrm{HbAlc}$ $6.0 \%-6.4 \%$. Compared with those with normal glucose tolerance, individuals with IGT; HR 1.13 , 95\% CI (1.05 to 1.21 ), IFG WHO; HR $1.14,95 \%$ CI (1.06 to 1.22$)$; IFG ADA; HR 1.10, 95\% CI (1.02 to 1.19) and HbA1c 5.7\%$6.4 \%$; HR 1.28 , $95 \%$ CI (1.13 to 1.46 ) had an increased risk for heart disease. There was no evidence of an increased risk of heart disease and HbAlc 6.0\%-6.4\% (online supplemental figure 6).

There were no significant differences in the association between prediabetes and heart disease by presence of a pre-existing CVD. The association between heart disease and HbA1c 5.7\%-6.4\% was increased in studies from the USA compared with those from Europe or Asia. There were no differences in the association of IGT, IFG ADA, IFG WHO or HbA1c $6.0 \%-6.4 \%$ and heart disease events by region.

We found no evidence of publication bias among studies assessing the association between heart disease and intermediate hyperglycemia based on visual inspection of the funnel plot (online supplemental figure 13).

\section{Intermediate hyperglycemia and incident heart failure, chronic kidney disease and retinopathy}

The association between intermediate hyperglycemia and heart failure was examined in five studies. Of these, four examined the association with IFG ADA, one examined the association with IGT and two examined the association with HbAlc 5.7\%-6.4\%. Neither IFG ADA; HR 0.92, $95 \%$ CI (0.76 to 1.12$)$ nor HbA1c $5.7 \%-6.4 \%$; HR 1.13 , $95 \%$ CI $(0.87$ to 1.18$)$ were associated with an increased risk of heart failure. IGT was moderately significantly associated with increased risk of heart failure; HR 5.04, 95\% CI (1.00 to 25.40) (online supplemental figure 7).

There were no significant differences in the association between prediabetes and incident heart failure by presence of pre-existing CVD or by region (online supplemental table 4). 
We found evidence of possible publication bias in studies examining the association between intermediate hyperglycemia based on visual inspection of the funnel plot (online supplemental figure 14).

The association between incident chronic kidney disease and intermediate glycemia was examined in eight studies. Of those, five studies examined the association with IGT, four examined the association with IFG ADA, five examined the association with IFG WHO, three examined the association with HbAlc ADA and one assessed the association between HbAlc IEC and chronic kidney disease. IGT was associated with an increased risk of chronic kidney disease; HR 1.09, 95\% CI (1.01 to 1.18). Both HbA1c 5.7\%-6.4\%; HR 1.32, $95 \%$ CI (1.16 to $1.50)$ and HbAlc $6.0 \%-6.4 \%$; HR $1.50,95 \%$ CI (1.32 to $1.70)$ were associated with an increased risk of chronic kidney disease. There were no significant associations with prediabetes as defined by the IFG ADA or IFG WHO criteria and chronic kidney disease (online supplemental figure 8).

All studies assessing the association between intermediate hyperglycemia and chronic kidney disease did so in participants with no history of baseline CVD. The association with IFG ADA and chronic kidney disease was increased in studies from the USA and the Middle East compared with those from Asia. The association between IFG WHO and chronic kidney disease was increased among studies from the USA compared with those from the Middle East or Asia. There were no significant differences in the association between intermediate hyperglycemia and chronic kidney disease by geographic region for either HbAlc criterion or IGT (online supplemental table 4).

There was some publication bias among studies assessing the association between chronic kidney disease and intermediate hyperglycemia based on visual inspection of the funnel plot (online supplemental figure 15).

Of the studies that met our inclusion criteria, one study examined the association between hyperglycemia and retinopathy for both IFG ADA and HbA1c ADA. Neither IFG ADA; HR $1.11,95 \%$ CI (0.84 to 1.45$)$ nor HbAlc $\mathrm{ADA}$; HR $0.84,95 \% \mathrm{CI}(0.61$ to 1.14$)$ were associated with incident retinopathy.

\section{DISCUSSION}

The ADA and WHO currently differ in their definitions of intermediate hyperglycemia, and it is unclear how well various definitions of intermediate hyperglycemia predict risk for mortality and cardiovascular events. In this meta-analysis including 106 articles from inception to January 31, 2020, which comprised cohorts from 27 countries, and included 1847523 individuals, we found that the current definition of IGT was associated with a $19 \%$ increased risk of all-cause mortality, a $21 \%$ increased risk of cardiovascular mortality, a $18 \%$ increased risk of incident cardiovascular events, a $13 \%$ increased risk in incident heart disease and a $24 \%$ increased risk in incident stroke compared with normal glucose tolerance.

With regard to fasting glycemia, IFG as defined by the WHO criteria $(110-125 \mathrm{mg} / \mathrm{dL})$ was associated with an $17 \%$ increased risk of all-cause mortality, a $20 \%$ increased risk of CVD mortality, a $21 \%$ increased risk in incident cardiovascular events, a $14 \%$ increased risk in incident heart disease and a 22\% increased risk in incident stroke. IFG as defined by the ADA criteria (100-125 mg/ $\mathrm{dL}$ ) was associated with an $11 \%$ increased risk of allcause mortality, a $14 \%$ increased risk for cardiovascular mortality, a $15 \%$ increased risk for cardiovascular events, an $10 \%$ increased risk for heart disease, a $7 \%$ increased risk for stroke and a $9 \%$ increased risk for chronic kidney disease.

HbA1c $6.0 \%-6.4 \%$ was associated with a $30 \%$ increased risk of all-cause mortality, a $32 \%$ increased risk of CVD and a $50 \%$ increased risk of chronic kidney disease. HbA1c $5.7 \%-6.4 \%$ was associated with a $15 \%$ increased risk in CVD events, a 28\% increased risk in heart disease, a $23 \%$ increased risk in stroke and a $32 \%$ increased risk in chronic kidney disease. There were not enough studies available to determine if prediabetes by any cut-point is significantly associated with increased risk of retinopathy

In recent years, a handful of meta-analyses have examined the associations between intermediate hyperglycemia and mortality or cardiovascular outcomes. A meta-analysis of 17 prospective cohort studies comprising 527021 individuals found that the risk of incident coronary heart disease was increased in those with IFG as defined by both the ADA and WHO criteria. ${ }^{16}$ Conversely, a meta-analysis of 15 studies including 760925 individuals noted that IGT or a combination of IFG and IGT were associated with a moderately elevated risk of stroke incidence. However, IFG defined by the ADA criteria alone was not. ${ }^{17} \mathrm{~A}$ previous meta-analysis conducted by Huang et al similarly examined the association between intermediate hyperglycemia ${ }^{18}$ and composite cardiovascular events and all-cause mortality, and noted that both IGT and IFG were associated with an increased risk of the outcomes of interest. A recently updated analysis of the Huang study by Cai et al examined the association between prediabetes and the risk of all-cause mortality and incident CVD in the general population and in patients with a history of atherosclerotic CVD in 10069955 individuals from 129 studies. ${ }^{19}$ Results of this study found that prediabetes was associated with an increased risk of all-cause mortality and CVD in both the general population and in patients with atherosclerotic CVD. ${ }^{19}$ Our study adds further evidence to support the notion that prediabetes is an increased risk state for mortality and cardiovascular events. We also found that prediabetes may be an increased risk for chronic kidney disease, particularly by the HbA1c ADA or HbAld IEC criteria.

Current recommendations to identify intermediate hyperglycemia are inconsistent and controversial. Both the WHO and ADA criteria for IFG are based on studies assessing the lower limits of fasting glucose above which 
the risk for developing diabetes increases considerably. ${ }^{20}$ The lower limit of IFG was originally set at $110 \mathrm{mg} / \mathrm{dL}$, which is still the cut-point accepted by $\mathrm{WHO},{ }^{2}$ and was based on an analysis of the Paris Prospective Study which reported that a fasting plasma glucose range between 110 and $126 \mathrm{mg} / \mathrm{dL}$ was similar to IGT in terms of predicting future diabetes prevalence. ${ }^{20}$ In 2003, the ADA lowered the cut-point for IFG to $100 \mathrm{mg} / \mathrm{dL}$ based on analyses of data from four populations: Pima Indian, Mauritius, San Antonio and Dutch. ${ }^{21}$ Results of these analyses indicated that $110 \mathrm{mg} / \mathrm{dL}$ was inappropriately high as the lower limit for IFG and suggested that a cut-point of $100 \mathrm{mg} / \mathrm{dL}$ would optimise the sensitivity and specificity for diabetes prediction. ${ }^{21}$ The results of our study found evidence of increased risk of all-cause mortality, CVD mortality, CVD, heart disease and stroke at both the IFG ADA and the IFG WHO cut-points.

In addition, the use of HbAlc to identify intermediate hyperglycemia is also controversial given that the ADA suggests a cut-point of HbA1c 5.7\%-6.4\% for intermediate hyperglycemia, while an IEC $^{4}$ as well as the UK-based National Institute for Health and Clinical Excellence $^{5}$ both recommend using HbA1c 6.0\%-6.4\%, and WHO currently does not offer any interpretation of HbAlc levels below $6.5 \%{ }^{22}$ In our study, we found that the higher cut-point of $6.0 \%-6.4 \%$ was associated with an increased risk of all-cause mortality, and chronic kidney disease, while the lower cut-point of 5.7\%-6.4\% was associated with an increased rsk of cardiovascular events, heart disease, stroke and chronic kidney disease. These results provide evidence to support the inclusion of HbAlc as a definition for prediabetes. However, few studies examined the relationship between $\mathrm{HbA1c} 6.0 \%$ $6.4 \%$ and mortality, cardiovascular outcomes, chronic kidney disease or retinopathy, and additional studies are needed to determine the association between HbAlc $6.0 \%-6.4 \%$ and adverse outcomes.

The results of our study should be interpreted in the context of limitations. All studies included were observational studies. Therefore, we cannot draw definitive causal associations, given that some studies may be subject to bias unaccounted for in the adjustment for confounding. There is also heterogeneity in the included study populations, follow-up times, outcome definitions and model specifications. In addition, many studies did not assess the isolated forms of IFG glucose, IGT or elevated HbA1c. Therefore, it is possible that studies assessing the risk of outcomes in individuals with IFG could also be including individuals with IGT or elevated HbAlc. Additionally, while we had data from 27 individual countries, the majority of studies came from Asia, Europe and the USA. Many regions, including the majority of South Asia, Africa and South and Central America were not represented. It is possible that the results would differ had there been more data from those regions. Lastly, the majority of the studies assessed the relationship between intermediate hyperglycemia and all-cause mortality, cardiovascular mortality, cardiovascular events or heart disease.
Additional research is needed to assess the strength of the association between intermediate hyperglycemia and heart failure, chronic kidney disease and retinopathy. However, our study also has several strengths. We included data from nearly 1.85 million individuals from 27 different countries, and were therefore able to assess the associations with varying types of prediabetes and the risk of mortality, CVD as well as microvascular outcomes such as chronic kidney disease. Lastly, the majority of the studies included in our analysis were of high quality and adequately adjusted for confounders.

In summary, the findings of our study note that IGT, as currently defined, adequately indicates increased risk for all-cause mortality, CVD mortality, incident cardiovascular events, incident heart disease and incident stroke. Furthermore, fasting glucose levels between 110 and $125 \mathrm{mg} / \mathrm{dL}$ as well as those between 100 and $125 \mathrm{mg} /$ $\mathrm{dL}$ were associated with an increased risk of mortality and cardiovascular events. However, compared with IFG ADA, IFG WHO was associated with an increased risk of all-cause mortality, CVD mortality, cardiovascular events, heart disease and stroke. Lastly, HbA1c levels 6.0\%$6.4 \%$ were associated with an increased risk of mortality and chronic kidney disease while HbAlc 5.7\%-6.4\% was associated with an increased risk of cardiovascular events, heart disease, stroke and chronic kidney disease. Our results indicate that prediabetes by IGT, IFG and HbAlc criteria is an increased risk state for mortality, cardiovascular and microvascular outcomes.

\section{Author affiliations}

${ }^{1}$ Rollins School of Public Health, Hubert Department of Global Health, Emory University, Atlanta, Georgia, USA

${ }^{2}$ Divisoin of Hospital Medicine, Emory University School of Medicine, Atlanta, Georgia, USA

${ }^{3}$ Department of Nutrition and Health Sciences, Laney Graduate School, Emory University, Atlanta, Georgia, USA

${ }^{4}$ Rollins School of Public Health, Department of Epdemiology, Emory University, Atlanta, Georgia, USA

${ }^{5}$ Department of Epidemiology and Biostatistics, George Washington University School of Public Health and Health Services, Washington, District of Columbia, USA

${ }^{6}$ Department of Political Science, Duke University, Durham, North Carolina, USA

Acknowledgements We would like to thank Hannah Rogers for her assistance with the literature search.

Contributors UPG contributed to the study inception and design, screening and extracting data, data interpretation and manuscript writing and editing. RJ was responsible for data screening and extraction, data analysis, data interpretation and manuscript editing. SH was responsible for data screening and extraction, the preparation and design of tables and figures and manuscript editing. $\mathrm{MH}$ was responsible for data screening and extraction, data analysis and manuscript editing. LRS and JW were responsible for data screening and extraction and manuscript editing. NS was responsible for data cleaning and organization. KMVN was responsible for study inception and design, data interpretation and manuscript editing. UPG had full access to all the data in the study and had final responsibility for the decision to submit for publication.

Funding UPG and KMVN were funded by the National Heart, Lung, and Blood Institute grant number R01HL125442. UPG and KMVN were funded by the National Institute of Diabetes and Digestive and Kidney Diseases grant number P30DK111024. UPG, KMVN, SH and MH were funded by the World Health Organization funding number 699062-0.

Map disclaimer The depiction of boundaries on the map(s) in this article does not imply the expression of any opinion whatsoever on the part of BMJ (or any member 
of its group) concerning the legal status of any country, territory, jurisdiction or area or of its authorities. The map(s) are provided without any warranty of any kind, either express or implied.

Competing interests None declared.

Patient consent for publication Not required.

Ethics approval Ethics approval was not required for this analysis.

Provenance and peer review Not commissioned; externally peer reviewed.

Data availability statement Data are available on reasonable request. All data relevant to the study are included in the article or uploaded as supplemental information.

Supplemental material This content has been supplied by the author(s). It has not been vetted by BMJ Publishing Group Limited (BMJ) and may not have been peer-reviewed. Any opinions or recommendations discussed are solely those of the author(s) and are not endorsed by BMJ. BMJ disclaims all liability and responsibility arising from any reliance placed on the content. Where the content includes any translated material, BMJ does not warrant the accuracy and reliability of the translations (including but not limited to local regulations, clinical guidelines, terminology, drug names and drug dosages), and is not responsible for any error and/or omissions arising from translation and adaptation or otherwise.

Open access This is an open access article distributed in accordance with the Creative Commons Attribution Non Commercial (CC BY-NC 4.0) license, which permits others to distribute, remix, adapt, build upon this work non-commercially, and license their derivative works on different terms, provided the original work is properly cited, appropriate credit is given, any changes made indicated, and the use is non-commercial. See: http://creativecommons.org/licenses/by-nc/4.0/.

ORCID iDs

Unjali P Gujral http://orcid.org/0000-0002-0352-813X

Lisa R Staimez http://orcid.org/0000-0002-2818-1187

K M Venkat Narayan http://orcid.org/0000-0001-8621-5405

\section{REFERENCES}

1 American Diabetes Association. Diagnosis and classification of diabetes mellitus. Diabetes Care 2014;37:S81-90.

2 World Health Organization, Federation ID. Definition and diagnosis of diabetes mellitus and intermediate hyperglycaemia: report of a WHO/IDF consultation. Geneva: World Health organization, 2006. Available: http://apps.who.int/iris/handle/10665/43588 [Accessed 27 Sep 2018].

3 Organization WH, others. Definition and diagnosis of diabetes mellitus and intermediate hyperglycaemia: report of a WH. published online first, 2006. Available: http://apps.who.int/iris/handle/10665/ 43588 [Accessed 31 May 2016].
4 International Expert Committee. International expert Committee report on the role of the A1c assay in the diagnosis of diabetes. Diabetes Care 2009;32:1327-34.

5 Type 2 diabetes: prevention in people at high risk / guidance and guidelines | NICE. Available: https://www.nice.org.uk/guidance/ ph38/chapter/Recommendations\#risk-assessment [Accessed 6 Nov 2018].

6 Abdul-Ghani MA, Tripathy D, DeFronzo RA. Contributions of -Cell Dysfunction and Insulin Resistance to the Pathogenesis of Impaired Glucose Tolerance and Impaired Fasting Glucose. Diabetes Care 2006;29:1130-9.

7 Færch K, Vaag A, Holst JJ, et al. Impaired fasting glycaemia vs impaired glucose tolerance: similar impairment of pancreatic alpha and beta cell function but differential roles of incretin hormones and insulin action. Diabetologia 2008:51:853-61.

8 Hayden JA, van der Windt DA, Cartwright JL, et al. Assessing bias in studies of prognostic factors. Ann Intern Med 2013;158:280.

9 Zhang J, KF Y. What's the Relative Risk?: A Method of Correcting the Odds Ratio in Cohort Studies of Common Outcomes. JAMA 1998;280:1690.

10 Yang Y, Li W, Zhu H, et al. Prognosis of unrecognised myocardial infarction determined by electrocardiography or cardiac magnetic resonance imaging: systematic review and meta-analysis. BMJ2020;369:m1184.

11 Higgins JPT, Thompson SG. Quantifying heterogeneity in a metaanalysis. Stat Med 2002;21:1539-58.

12 DerSimonian R, Laird N. Meta-Analysis in clinical trials. Control Clin Trials 1986;7:177-88.

13 Viechtbauer W, Cheung MW-L. Outlier and influence diagnostics for meta-analysis. Res. Synth. Method 2010;1:112-25.

14 Schwarzer G, Schwarzer MG. Package 'meta.'. In: The $R$ foundation for statistical computing. 9, 2012.

15 Viechtbauer W. Conducting meta-analyses in R with the metafor package. J Stat Softw 2010;36:1-48

$16 \mathrm{Xu} \mathrm{T}$, Liu W, Cai X, et al. Risk of coronary heart disease in different criterion of impaired fasting glucose. Medicine 2015;94:e1740.

17 Lee M, Saver JL, Hong K-S, et al. Effect of pre-diabetes on future risk of stroke: meta-analysis. BMJ2012;344:e3564.

18 Huang Y, Cai X, Mai W, et al. Association between prediabetes and risk of cardiovascular disease and all cause mortality: systematic review and meta-analysis. BMJ 2016;355:i5953.

19 Cai X, Zhang Y, Li M. Association between prediabetes and risk of all cause mortality and cardiovascular disease: updated meta-analysis. BMJ2020;370.

20 Charles MA, Fontbonne A, Thibult N, et al. Risk factors for NIDDM in white population: Paris prospective study. Diabetes 1991;40:796-9.

21 Kahn R. Follow-Up report on the diagnosis of diabetes mellitus: the expert Committee on the diagnosis and classifications of diabetes mellitus*. Diabetes Care; Alexandria 2003;26:3160-7.

22 Organization WH. Use of glycated haemoglobin ( $\mathrm{HbA1C}$ ) in the diagnosis of diabetes mellitus. Abbreviated report of a who consultation 2011. Geneva: World Health Organization, 2013. 